\title{
Evaluation Of Oral Health-Related Quality Of Life In Children With Hematological Malignances
}

\author{
munevver kilic ${ }^{1}$ and Sevcihan Gunen Yilmaz ${ }^{2}$ \\ ${ }^{1}$ Ataturk Universitesi \\ ${ }^{2}$ Akdeniz Universitesi Dis Hekimligi Fakultesi
}

September 10, 2021

\begin{abstract}
Objective: Chemotherapy and radiotherapy, used to treat childhood hematological malignancies (HM), can negatively impact oral tissues and organs. This study aimed to evaluate oral health-related quality of life in children with HM. Material and Methods: A total of 99 children, including 49 undergoing HM (41 for acute lymphocytic leukemia, 8 for acute myelocytic leukemia,) and 50 healthy volunteers, were included in this cross-sectional study. The mean age of the entire study group was $78.63 \pm 34.41$ months. The mean age of the HM and control groups was $87.12 \pm 35.04$ and $70.95 \pm 34.85$ months, respectively. The Simplified Oral Hygiene Index (SOHI), Decayed, Missing, and Filled Teeth (DMFT/dmft) index, and Turkish version of the Early Childhood Oral Health Impact Scale (ECOHIS-T) were administered to all children. The data were analyzed using SPSS software (version 22.0). Results: The age and gender distributions of the two groups were similar. The SOHI was significantly higher in the HM group, whereas the DMFT/dmft score was similar between the groups. No significant difference in the total ECOHIS-T score was observed between the two groups, but there was a group difference in the responses to questions on pain and psychological processes. Conclusions: Oral health and self-care were negatively affected by childhood HM and the treatment thereof. Close clinical dental follow-up of such patients is required.
\end{abstract}

\section{INTRODUCTION}

Hematological malignancies (HM) account for approximately $40 \%$ of all childhood malignancies ${ }^{1}$. Leukemias constitute the vast majority of childhood HM, and the most common of these is acute lymphoblastic leukemia (ALL) ${ }^{2}$.Hematopoietic stem cell transplantation, chemotherapy (CT), and radiotherapy (RT) are widely used treatments that increase the survival rates of children with HM. CT and RT are first-line treatments, but they have acute and chronic effects on many tissues and organs, which may decrease overall life expectancy and quality of life $(\mathrm{QoL})^{3}$. Oral complications, such as mucositis, oral ulcerations, bleeding, hematoma, dysphagia, malnutrition, and infection, which may be life-threatening and affect survival, can occur depending on the nature of the disease, treatment, and existing oral health problems ${ }^{4}$. Oral health problems negatively affect both children and their parents ${ }^{5}$. The oral hygiene status of a child with HM, and the education level and socioeconomic status of the parents, affect the survival and QoL of patients ${ }^{6}$. Evaluating oral health, and preventing and treating oral complications, can improve oral function and QoL, and reduce morbidity ${ }^{7}$. Oral complications during cancer treatment can interrupt the treatment or decrease its effectiveness. Pediatric dentists play an important role in the management of these life-threatening diseases ${ }^{8}$.

This study aimed to determine the oral health-related quality of life (OHRQoL) of children with HM who underwent RT and CT examinations for various reasons.

\section{MATERIAL AND METHODS}

\subsection{Study Design}


This study used a single-center cross-sectional design. We enrolled 49 pediatric patients with HM (Group 1) and 50 healthy volunteers (Group 2), who presented to Atatürk University Faculty of Dentistry Department of Pedodontics between 2018 and 2020 for oncological treatment. Children with acute myelocytic leukemia (AML), ALL, were included in the HM group. Children with malign melanoma, hodgkin lymphoma or nonhodgkin lymphoma, were excluded in the HM group because the number of children with these cancers was low. Healthy children presenting to the clinic for various reasons were included in the control group. The children in the control group did not have any systemic disease.

\subsection{Data Collection}

Demographic (age, gender, and parental education status), Early Childhood Oral Health Impact Scale (ECOHIS) and QoL data were evaluated. The dentition period and dental occlusion status were determined by an intraoral examination, and the Decayed, Missing, and Filled Teeth (DMFT/dmft) index was applied along with the Simplified Oral Hygiene Index (SOHI).

2.3 SOHI: Six teeth, considered representative of all anterior and posterior teeth, were evaluated (numbers $16,26,11,31,36$, and 46). The facial surfaces of teeth 16, 26, 11, and 31, and the lingual surfaces of teeth 36-46, were also evaluated. After obtaining debris and calculus scores on a 4-point (0-3) scale, the SOHI was administered.

2.4 DMFT index: The decayed, filled, and lost teeth of the participants were identified, and the data were recorded based on the DMFT/dmft scale (DT: decayed teeth, MT: lost teeth, FT: filled teeth, dt: decayed primary teeth, mt: lost primary teeth, ft: filled primary teeth).

2.5 ECOHIS-T: This scale consists of 13 questions answered using 4-point Likert scales (14). The data were recorded by a single pedodontist. The first nine and final four questions comprise the child impact scale (CIS) and family impact scale (FIS), respectively. The CIS covers four domains and the FIS two domains. The CIS includes one question on symptoms, four on function, two on psychological processes, one on self-perception, and one on social communication. The FIS consists of two questions each on parental distress and family function. Each question is scored between 0 and 5; total scores therefore range between 0 and 52 (0-36 CIS and $0-16$ for FIS) (15).

\subsection{Ethics Approval}

The Clinical Research Ethics Committee of the Erzurum Regional Training and Research Hospital approved this study (2021 / 05-96), which was carried out according to the Declaration of Helsinki.

\subsection{Statistical Analysis}

The statistical analysis was conducted using SPSS for Windows software (version 22.0; IBM Corp., Armonk, NY, USA). The intraobserver coefficient of variation for all assessments was 0.94 . Normality tests were performed for all variables. Age, gender, parental education status, dentition period, occlusion type, and DMFT/dmft and ECOHIS-T results were compared between the HM and control groups using the MannWhitney $U$ test. Correlations between the SOHI, DMFT/dmft, and ECOHIS-T scores were evaluated using Spearman's rank correlation analysis. A p-value $<0.05$ was considered significant.

\section{RESULTS}

A total of 99 children were included, of whom 49 (25 girls and 24 boys) were in the HM group and 50 (24 girls and 26 boys) were healthy volunteers. The mean age of the entire study group was $78.63 \pm 34.41$ months. The mean age of the HM and control groups was $87.12 \pm 35.04$ and $70.95 \pm 34.85$ months, respectively (p $=0.08)$. No significant gender difference was observed between the groups $(\mathrm{p}=0.70)$ Of the children in the HM group, 41 were diagnosed with ALL, 8 with AML. No significant difference in the parental education level was observed between the groups $(\mathrm{p}=0.12)$ The mean DMFT/dmft score was $5.76 \pm 5.21$ and $7.27 \pm$ 4.98 in the control and HM groups, respectively $(\mathrm{p}=0.22)$. Regarding the dentition period, $43.3 \%$ of the children in the control group had deciduous dentition and $53.7 \%$ had mixed dentition. A total of $26.1 \%$ of the children in the HM group had deciduous dentition, $55.1 \%$ had mixed dentition, and $34.4 \%$ had permanent 
dentition $(\mathrm{p}=0.02)$. Regarding the occlusion type, $56 \%$ of the control group had Class 1 closure, compared to $95 \%$ of the HM group ( $\mathrm{p}=0.01)$. According to the SOHI, $46.7 \%, 30 \%$ and $23.3 \%$ of the control group had good, poor and bad oral hygiene, respectively, compared to $24.1 \%, 31 \%$, and $44.8 \%$ of the HM group. Oral hygiene was significantly better in the control than HM group $(\mathrm{p}=0.04)$ (Table 1).

No significant difference in total ECOHIS score was observed between the groups. Children in the HM group had significantly more mouth-jaw pain than those in the control group according to the ECOHIS-T $(\mathrm{p}=$ 0.05). No significant differences in eating hot and cold foods, drinking, speaking, or school attendance were observed between the HM and control groups $(\mathrm{p}=0.60,0.13,0.40$, and 0.15 , respectively). No significant differences in scores on the items pertaining to psychological processes, self-perception and social interaction (e.g., falling asleep due to dental pain and treatment, irritability/restlessness, and fear of talking) were observed between the groups $(\mathrm{p}=0.87,0.94$, and 0.64 , respectively). Hesitancy to smile and laugh was significantly greater in the HM than in control group $(\mathrm{p}=0.035)$. No significant differences in discomfort caused by dental problems and treatments, feeling guilty, taking a break from work, or financial problems were observed between the groups $(\mathrm{p}>0.05)$ (Table 2).

The DMFT/dmft score was highly correlated with the SOHI and ECOHIS-T scores (Spearman's rank correlation coefficient $=0.707$ and 0.721 , respectively; Table 3 ).

\section{DISCUSSION}

Leukemias constitute the vast majority of childhood HM, and the most common of these is ALL ${ }^{9}$. In this study, the majority of the children with HM were ALL patients, which agrees with previous studies. Oral health can adversely affect the QoL of adults and children, and their families. ${ }^{10}$ The SOHI has been used to obtain information about the oral care habits and QoL of individuals varying in age, ethnicity, and sociocultural and health status ${ }^{11}$. In this study, the SOHI was higher in the HM group than the control group, despite similar sociocultural and economic conditions; the difference may have been related to treatment complications, such as mucositis, saliva volume and swallowing difficulties, along with poorer self-care in children with $\mathrm{HM}^{12,13}$.OHRQoL is an important public health issue with physical and psychological implications. OHRQoL differs among communities and ethnic groups, depending on the level of awareness of its importance and access to treatment ${ }^{14}$.

OHRQoL research has shown utility in studies of diverse populations, including oral cancer patients ${ }^{15}$, sleep apnea patients ${ }^{16}$, toddlers with early childhood caries ${ }^{17}$, and children with craniofacial anomalies ${ }^{18}$.

Various scales, such as the ECOHIS ${ }^{19}$ and Child Perceptions Questionnaire ${ }^{20}$, have been used to determine OHRQoL in children of various ages from different countries and communities. In particular, the ECOHIS questionnaire has been widely used to assess the impact of several oral problems on QoL; it has been frequently been applied in studies of preschool children, and is suitable and reliable for assessing preschool and elementary school students ${ }^{21}$. The original ECOHIS assessed the impact of oral health problems, and the treatments used to address them, on the QoL of preschool children and their families ${ }^{21}$.

The ECOHIS was originally developed in English to determine OHRQoL in preschool children, but has since been translated into different languages and validated for use in various cultural settings ${ }^{5}$. The ECOHIS-T has acceptable validity and reliability ${ }^{22}$.

The ECOHIS has been used for aesthetic and functional evaluations of oral tissues, malocclusion ${ }^{23}$, dental trauma, cerebral palsy ${ }^{24}$, autism spectrum disorder ${ }^{25}$, bruxism ${ }^{26}$, children with cleft lip and palate, and HIV-positive and negative children ${ }^{27}$. It has also been used to assess the effects of maternal depression and anxiety on pediatric OHRQoL ${ }^{28}$, and to evaluate oral health in patients with head and neck cancers. Rodrigues et al. ${ }^{16}$ detected a negative correlation between OHRQoL evaluated by the ECOHIS and bruxism, while Alencar et al. ${ }^{26}$ reported that this correlation was mediated by anxiety. In a study conducted by Du et al. ${ }^{25}$, a significant difference was observed in the ECOHIS scores of children with and without cerebral palsy and autism spectrum disorder. Birunghi et al. ${ }^{27}$ detected a significant difference in the ECOHIS score between children with and without HIV. Costa et al. ${ }^{28}$ showed that the OHRQoL of mothers with maternal 
depression and anxiety was lower compared to healthy mothers. In the present study, no significant difference was found in total OHRQoL scores between healthy children and those with HM.The incidence of pain was closer to that reported in other validation studies, which ranged from 15 to $24 \%^{13}$. The rate of pain in the control and HM groups ranged from 20.1 to $44.8 \%$ and was significantly higher in the HM group than the control group. While the incidence of pain in the control group was comparable with the literature, the higher incidence in our HM children compared to previous studies may be associated with differences in the oral complications of treatments or pain perception of the patients, or with the effect of disease on oral tissues $^{13}$.

$\mathrm{Du}$ et al. $^{25}$ conducted a study on healthy children with cerebral palsy and reported significant differences on all questions, including ones about self-image and social interactions on the CIS of the ECOHIS. In the present study, hesitancy to smile, and issues with self-perception and social interaction, were observed significantly more frequently in the HM than control group.

Khari et al. ${ }^{29}$ reported that ECOHIS scores were negatively correlated with parental education level. Similarly, in the present study, education level and the ECOHIS and DMFT/dmft scores were negatively correlated.

The DMFT/dmft has been used to determine oral health in children and adults with type 1 diabetes mellitus ${ }^{30}$, HIV-positive adults, and children receiving antineoplastic treatment ${ }^{31}$, as well as in studies of fetal alcohol syndrome $^{32}$, ALL and asthma ${ }^{33}$, orthodontic anomalies ${ }^{34}$, prosthodontic problems ${ }^{35}$, patients with a short dental $\operatorname{arch}^{35}$, patients with high sugar intake ${ }^{36}$, and tooth brushing habits ${ }^{37}$.

Dubey et al. reported that DMFT/dmft scores were higher in children with ALL than in those with type 1 diabetes mellitus or asthma ${ }^{33}$.

Olczak-Kowalczyk et al. showed that the DMFT/dmft scores during the post-treatment period are higher than those in children aged 5-18 years during and after antineoplastic $\mathrm{CT}^{38}$. In the current study, the DMFT/dmft scores were similar between the healthy and HM groups. This result may be associated with late complications of antineoplastic treatments and a decrease in patient self-care skills.

When there is a normal occlusal relationship, the primary dentition are parallel to the permanent dentition. The occlusal relationships of teeth vary during the primary dentition period and are important for early diagnosis and treatment of malocclusion ${ }^{39}$. Dentition and dental occlusion status are important oral health parameters for both milk and permanent teeth. In the current study, the HM group had significantly more advanced dentition compared to the control group, and a higher incidence of Class 1 dental occlusion. Although this is advantageous for maintaining good oral health, in this study the difference may have been due to the age difference between the control and HM groups.

This study had some limitations. First, it was cross-sectional and certain parameters in the HM group could not be evaluated both before and after treatment. Second, a limited number of patients were included, and they were all from the same center. Third, the types and doses of CT contrast agents were unknown, as were the RT dose schedules.

\section{CONCLUSION}

Improving oral health, and thus OHRQoL, is functionally and psychologically important for children with $\mathrm{HM}$, and for their parents.

\section{CLINICAL RELAVANCE}

\subsection{Scientific rationale of the study}

Oral health of children with hematological malignancies are curicial factor in maintaining general health . This study also indicates the effect of oral health on quality of life in children with hematological malignancies.

\subsection{Principal findings}


Oral hygiene was significantly better in the control than HM group.

\subsection{Practical implications}

Children with hematological malignancies can improve their quality of life if they are under the control of a dentist together with the diagnosis.

\section{REFERENCES}

1. Ries LAG. Cancer incidence and survival among children and adolescents: United States SEER program, 1975-1995 . National Cancer Institute; 1999.

2. Lohi O, Kanerva J, Taskinen M, et al. Lapsuusian leukemia. 2013;

3. Bevans M, El-Jawahri A, Tierney DK, et al. National Institutes of Health hematopoietic cell transplantation late effects initiative: the patient-centered outcomes working group report. Biol Blood Marrow Transplant . 2017;23(4):538-551.

4. Francisconi CF, Caldas RJ, Oliveira Martins LJ, Fischer Rubira CM, da Silva Santos PS. Leukemic oral manifestations and their management.Asian Pac J Cancer Prev . 2016;17(3):911-915.

5. Farsi NJ, El-Housseiny AA, Farsi DJ, Farsi NM. Validation of the Arabic version of the early childhood oral health impact scale (ECOHIS). BMC Oral Health . 2017;17(1):1-11.

6. Mogensen H, Modig K, Tettamanti G, Erdmann F, Heyman M, Feychting M. Survival after childhood Cancer-social inequalities in high-income countries. Front Oncol . 2018;8:485.

7. Ribeiro ILA, Limeira RRT, Dias de Castro R, Ferreti Bonan PR, Valenca AMG. Oral mucositis in pediatric patients in treatment for acute lymphoblastic leukemia. Int J Environ Res Public Health . 2017;14(12):1468.

8. Kapoor G, Goswami M, Sharma S, Mehta A, Dhillon JK. Assessment of oral health status of children with Leukemia: A cross-sectional study.Spec Care Dentist . 2019;39(6):564-571.

9. Brayley J, Stanton LK, Jenner L, Paul SP. Recognition and management of leukaemia in children. $B r J$ Nurs . 2019;28(15):985-992.

10. Nasu D, Uematsu A, Nakamura S, et al. Oral hygiene and oral status of institutionalized children with motor and intellectual disabilities.J Oral Sci . 2020;62(1):89-92.

11. Vpk V, Mohanty VR, Balappanavar AY, Juneja M, Gupta V, Kapoor S. Effectiveness of different parenting interventions on oral hygiene of cerebral palsy children: A randomized controlled trial. Spec Care Dentist . 2020;40(4):335-343.

12. Hamzavi SS, Amanati A, Badiee P, et al. Changing face of Candida colonization pattern in pediatric patients with hematological malignancy during repeated hospitalizations, results of a prospective observational study (2016-2017) in shiraz, Iran. BMC Infect Dis . 2019;19(1):1-9.

13. Epstein JB, Thariat J, Bensadoun RJ, et al. Oral complications of cancer and cancer therapy: from cancer treatment to survivorship. CA: Cancer J Clin . 2012;62(6):400-422.

14. Neelakantan P, Liu P, Dummer PM, McGrath C. Oral health-related quality of life (OHRQoL) before and after endodontic treatment: a systematic review. Clinic Oral Investig . 2020;24(1):25-36.

15. Ship JA. Oral health-related quality of life in patients with oral cancer. Oral health-related quality of life . 2002:153-168.

16. Rodrigues JA, Azevedo CB, Chami VO, Solano MP, Lenzi TL. Sleep bruxism and oral health-related quality of life in children: a systematic review. Int J Paediatr Dentist . 2020;30(2):136-143.

17. Filstrup SL, Briskie D, da Fonseca M, Lawrence L, Wandera A, Inglehart MR. Early childhood caries and quality of life: child and parent perspectives. Pediatr Dentist . 2003;25(5):431-440. 
18. Broder HL. International confederation for cleft lip and palate and related craniofacial anomalies task force report: Holistic Outcomes. Cleft Palate Craniofac J; 2014;51(6):130-4.

19. Pahel BT, Rozier RG, Slade GD. Parental perceptions of children's oral health: the Early Childhood Oral Health Impact Scale (ECOHIS). Health Qual Life Outcomes . 2007;5(1):1-10.

20. Sun L, Wong HM, McGrath CP. The factors that influence the oral health-related quality of life in 12year-old children: baseline study of a longitudinal research. Health Qual Life Outcomes . 2017;15(1):1-14.

21. Rando GM, Jorge PK, Vitor LLR, et al. Oral health-related quality of life of children with oral clefts and their families. J Appl Oral Sci . 2018;26

22. Peker K, Uysal O, Bermek G. Cross-cultural adaptation and preliminary validation of the Turkish version of the Early Childhood Oral Health Impact Scale among 5-6-year-old children. Health Qual Life Outcomes . 2011;9(1):1-11.

23. Sousa RV, Clementino MA, Gomes MC, Martins CC, Granville-Garcia AF, Paiva SM. Malocclusion and quality of life in B razilian preschoolers.Eur J Oral Sci . 2014;122(3):223-229.

24. Du RY, McGrath C, Yiu CK, King NM. Health-and oral health-related quality of life among preschool children with cerebral palsy. Qual Life Res . 2010;19(9):1367-1371.

25. Du R, Yiu C, King N. Health-and oral health-related quality of life among preschool children with autism spectrum disorders. Eur Arch Paediatr Dent . 2020;21(3):363-371.

26. de Alencar NA, Leao CS, Leao ATT, Luiz RR, Fonseca-Goncalves A, Maia LC. Sleep bruxism and anxiety impacts in quality of life related to oral health of Brazilian children and their families. $J$ Clin Pediatr Dent . 2017;41(3):179-185.

27. Birungi N, Fadnes LT, Engebretsen IM, et al. Caries experience and oral health related quality of life in a cohort of Ugandan HIV-1 exposed uninfected children compared with a matched cohort of HIV unexposed uninfected children. BMC Public Health . 2020;20:1-12.

28. Costa FdS, Azevedo MS, Ardenghi TM, Pinheiro RT, Demarco FF, Goettems ML. Do maternal depression and anxiety influence children's oral health-related quality of life? Community Dent Oral Epidemiol . 2017;45(5):398-406.

29. Khatri SG, Acharya S, Srinivasan S. Mothers' sense of coherence and oral health related quality of life of preschool children in Udupi Taluk. Community dental health . 2014;31(1):32-36.

30. Rafatjou R, Razavi Z, Tayebi S, Khalili M, Farhadian M. Dental health status and hygiene in children and adolescents with type 1 diabetes mellitus. J Res Health Sci . 2016;16(3):122.

31. Wang Y, Zeng X, Yang X, et al. Oral health, caries risk profiles, and oral microbiome of pediatric patients with leukemia submitted to chemotherapy. Biomed Res Int 2021:6637503.

doi: $10.1155 / 2021 / 6637503$

32. Blanck-Lubarsch M, Dirksen D, Feldmann R, Sauerland C, Hohoff A. Tooth malformations, DMFT index, speech impairment and oral habits in patients with fetal alcohol syndrome. Int $J$ Environ Res Public Health . 2019;16(22):4401.

33. Dubey S, Saha S, Tripathi AM, Bhattacharya P, Dhinsa K, Arora D. A comparative evaluation of dental caries status and salivary properties of children aged 5-14 years undergoing treatment for acute lymphoblastic leukemia, type I diabetes mellitus, and asthma-In vivo.J Indian Soc Pedod Prev Dent 2018;36(3):283.

34. Kukletova M, Izakovicova Holla L, Musilova K, Broukal Z, Kukla L. Relationship between gingivitis severity, caries experience and orthodontic anomalies in 13-15 year-old adolescents in Brno, Czech Republic. Community Dent Health .2012;29(2):179. 
35. Zhang Q, Kreulen CM, Witter DJ, Creugers NH. Oral health status and prosthodontic conditions of Chinese adults: a systematic review.Int $J$ Prosthodont . 2007;20(6)

36. Hong J, Whelton H, Douglas G, Kang J. Consumption frequency of added sugars and UK children's dental caries. Community Dent Oral Epidemiol . . 2018;46(5):457-464.

37. Gibson S, Williams S. Dental caries in pre-school children: associations with social class, toothbrushing habit and consumption of sugars and sugar-containing foods. Caries Res . 1999;33(2):101-113.

38. Olczak-Kowalczyk D, Krasuska-Sławińska E, Brożyna A, Turska-Szybka A, Dembowska-Bagińska B. Dental caries in children and adolescents during and after antineoplastic chemotherapy. J Clin Pediatr Dent . 2018;42(3):225-230.

39. Nanda RS, Khan I, Anand R. Age changes in the occlusal pattern of deciduous dentition. J Dent Res . 1973;52(2):221-224.

\section{Hosted file}

TABLES 1.docx available at https://authorea.com/users/430214/articles/533742-evaluation-oforal-health-related-quality-of-life-in-children-with-hematological-malignances

\section{Hosted file}

tables 2.docx available at https://authorea.com/users/430214/articles/533742-evaluation-oforal-health-related-quality-of-life-in-children-with-hematological-malignances

\section{Hosted file}

TABLES. 3docx.docx available at https://authorea.com/users/430214/articles/533742-evaluationof-oral-health-related-quality-of-life-in-children-with-hematological-malignances 